\title{
Randomized Controlled Trial of Intravenous Dexmedetomidine for Control of Hemodynamics, Surgical Bleeding, and Recovery Profile during Transsphenoidal Pituitary Surgery
}

\author{
Preethy J. Mathew ${ }^{1}$ Aparna Jain ${ }^{1} \quad$ Jyotsna Wig ${ }^{1} \quad$ Kanchan K. Mukherjee $^{2} \quad$ Aakriti Gupta $^{1}$
}

\footnotetext{
${ }^{1}$ Department of Anaesthesia and Intensive Care,

Post Graduate Institute of Medical Education and Research, Chandigarh, India

${ }^{2}$ Department of Neurosurgery, Post Graduate Institute of Medical Education and Research, Chandigarh, India
}

\author{
Address for correspondence Aakriti Gupta, MD, Department \\ of Anesthesia and Intensive Care, Post Graduate Institute of \\ Medical Education and Research, Chandigarh 160012, India \\ (e-mail: aakriti1988.ag@gmail.com).
}

\begin{abstract}
Keywords

- transsphenoidal pituitary surgery

- dexmedetomidine

- hemodynamics

Background Microscopic transsphenoidal excision of pituitary gland, though considered safe from neurosurgical point of view, produces marked cardiovascular and stress responses despite adequate depth of anesthesia. The aim of this study was to evaluate the effect of dexmedetomidine bolus and infusion in microscopic transsphenoidal pituitary surgery on hemodynamics, bleeding in surgical field, and recovery parameters.

Materials and Methods A prospective randomized double-blind study was conducted in 40 American Society of Anesthesiologists (ASA) I-II patients between 18 and 65 years, who underwent elective transsphenoidal excision of pituitary tumor. Two groups were constituted: one group received dexmedetomidine $1 \mu \mathrm{g} / \mathrm{kg}$ loading dose followed by $0.5 \mu \mathrm{g} / \mathrm{kg} / \mathrm{h}$ infusion, the other received placebo bolus and infusion. A standard anesthesia protocol including isoflurane and fentanyl was used in both groups. The hemodynamic profile, intraoperative bleeding, and recovery profile were studied.

Results The heart rate (HR) did not vary significantly from the baseline during surgery and recovery in the dexmedetomidine group. The systolic, diastolic, and mean blood pressures decreased significantly with dexmedetomidine in statistical terms, though they were within the clinically acceptable range and did not require intervention. On comparing the groups, dexmedetomidine group had better perioperative blood pressure profile and decreased isoflurane requirement. Bleeding scores during surgery and recovery were similar in both groups.

Conclusions Intraoperative use of dexmedetomidine in transsphenoidal pituitary surgery provided stable perioperative hemodynamics comprising reduced cardiovascular response to intubation and surgical noxious stimuli along with a similar recovery compared with the control group. Therefore, dexmedetomidine appears as a useful adjunct to control hemodynamics in neurosurgical patients undergoing transsphenoidal surgery.
\end{abstract}

DOI https://doi.org/

$10.1055 / \mathrm{s}-0039-1697558$

ISSN 2348-0548.
(C2020 Indian Society of Neuroanesthesiology and Critical Care
License terms

(ㅇ)(1) $\Theta \circledast$ 


\section{Introduction}

Transsphenoidal microsurgical excision of the pituitary tumor is often marked by cardiovascular and stress responses despite adequate depth of anesthesia. ${ }^{1,2}$ Conventional techniques employed to stabilize hemodynamics include narcotic analgesics, deep plane of inhalational anesthesia, local anesthetic infiltration, adrenoreceptor blockers, and vasodilator agents. ${ }^{1,3}$ However, each of these methods is fraught with its own complications, and the ideal method for obtunding the stress response during the surgery has remained elusive.

Dexmedetomidine has shown promise in recent years to confer stable hemodynamics and reduced surgical bleeding in endoscopic procedures. ${ }^{4,5}$ Few authors have studied the potential advantages of dexmedetomidine in transsphenoidal pituitary surgery, however, with contrasting findings. ${ }^{6,7}$ Therefore, we performed a randomized controlled study to evaluate the effect of dexmedetomidine infusion on intraoperative hemodynamics, surgical field bleeding, and recovery profile in patients undergoing transsphenoidal pituitary surgery under inhalational anesthesia.

\section{Materials and Methods}

After approval from institutional ethics committee and written informed consent from patients, 40 ASA I-II patients between 18 and 65 years of age, scheduled for elective transsphenoidal pituitary surgery were included in this prospective randomized double-blind study. The trial was registered with the Clinical Trial Registry of India (CTRI/2018/03/012630). The patients with decreased level of consciousness, increased intracranial pressure, risk of impending cerebral herniation, anticipated elective postoperative ventilation, coexisting intracranial aneurysms, or arteriovenous malformations were excluded. Similarly, patients with significant cardiovascular comorbidity, severe respiratory disease, and those with body weight more than $130 \%$ of ideal body weight, were also excluded.

Patients were allocated into two equal groups-group D for dexmedetomidine and group P for placebo-using computer-generated random number table in sequentially numbered opaque envelopes. On arrival in the operating room, monitors were attached to the patients including continuous electrocardiography, pulse oximetry, bispectral index (BIS), noninvasive blood pressure, and neuromuscular monitoring. Patients in group D received intravenous (IV) dexmedetomidine $1 \mu \mathrm{g} / \mathrm{kg}$ over a 10-minute period before induction of anesthesia and $0.5 \mu \mathrm{g} / \mathrm{kg} / \mathrm{h}$ by continuous IV infusion during the procedure. Group P received an equivalent volume of normal saline as placebo for IV bolus and maintenance infusion. The drugs were prepared by an independent anesthesiologist who was neither involved in anesthetic management nor outcome assessment.

After administration of study drug, fentanyl $1 \mu \mathrm{kg} / \mathrm{kg}$ IV was administered. Anesthesia was induced with propofol until BIS reached 50 and neuromuscular blockade was achieved with vecuronium IV bolus, followed by intermittent doses while maintaining train-of-four (TOF) count of 0 to 1 (NMT MechanoSensor; Datex-Ohmeda, Helsinki, Finland). Anesthesia was maintained with nitrous oxide and oxygen (50:50) in isoflurane to maintain BIS at 40 to 60 , and lungs were ventilated mechanically to ensure end-tidal carbon dioxide $\left(\mathrm{EtCO}_{2}\right)$ at 30 to $35 \mathrm{~mm} \mathrm{Hg}$.

Intranasal mucosal injection of 10 to $15 \mathrm{~mL}$ of lignocaine containing adrenaline $(1: 200,000)$ was used to prepare the nasal passages. Intraoperative fentanyl bolus $0.5 \mu \mathrm{g} / \mathrm{kg}$ IV was administered whenever BP or HR increased to $>20 \%$ of baseline. Isoflurane anesthesia and dexmedetomidine infusion were discontinued approximately 10 minutes before the estimated end of surgery. The patients were allowed to recover spontaneously until the return of TOF ratio $\left(\mathrm{T}_{1} / \mathrm{T}_{4}=25 \%\right)$. Neostigmine $0.05 \mathrm{mg} / \mathrm{kg}$ and glycopyrrolate $0.01 \mathrm{mg} / \mathrm{kg}$ was administered IV to antagonize residual neuromuscular blockade and tracheal extubation was performed after return of consciousness and neuromuscular power.

\section{Data Collection}

All the intraoperative and postoperative observations were recorded by a blinded observer. Hemodynamics were recorded. Blood loss was estimated by the operating surgeon who was blinded to randomization sequence on two scales: (1) Intraoperative bleeding score (IBS) ${ }^{4}$ every 10 minutes: 0 no bleeding (excellent surgical conditions), 1 minimum bleeding (sporadic suction), 2 diffuse bleeding (repeated suction), and 3 considerable bleeding (continuous suction). (2) Final opinion on bleeding score (FOBS $)^{4}$ at the end of surgery on a five-point scale ( 1 very low, 2 low, 3 average, 4 high, and 5 very high). The mean value of IBS for each patient was calculated as the mean of all values measured every 10 minutes. The other parameters recorded were: (1) time to extubation of trachea (interval from discontinuation of anesthetic agent to extubation), (2) time to response to verbal commands from discontinuation of anesthetic agent (assessed by asking the patient to open eyes or to squeeze the observer's hand), and (3) orientation time from discontinuation of anesthetic agent (assessed by asking the patient to tell his name and the place where he or she is). Modified Aldrete's Post Anesthesia Recovery Scoring system ${ }^{8}$ and Ramsay sedation score ${ }^{9}$ were assessed at 0,1 , and 2 hours.

\section{Statistical Analysis}

Sample size calculation revealed that 20 patients in each group were required to detect a difference of $10 \mathrm{~mm} \mathrm{Hg}$ in mean arterial pressure (MAP) with $\alpha$ error of 0.05 and $\beta$ error of 0.2 . The primary outcome measure was change in cardiovascular responses during the surgery. The secondary outcome measures were: bleeding in the surgical field, isoflurane requirement, and recovery parameters. Changes in hemodynamic variables within the group were analyzed by repeated measures analysis of variance (ANOVA) and paired $t$-test. Changes in hemodynamic variables between the groups were analyzed by one-way ANOVA and post hoc test (Turkey's HSD). Isoflurane and recovery parameters were analyzed using independent $t$-test. Bleeding scores were analyzed 
using Mann-Whitney test. Chi-square test and Fisher's exact test were used for categorical data. The $p$-value $<0.05$ was considered as statistically significant. SPSS v.15 (Chicago, Illinois, United States) was used for statistical analysis.

\section{Results}

Forty patients were enrolled in the study. The CONSORT flow diagram is presented in - Fig. 1. The clinical and demographic data of patients in both the groups were similar. Heart rate did not change significantly from baseline in group D, whereas it increased significantly in group $P$ after extubation $(p<0.05 ;-$ Fig. 2A). MAP decreased significantly from baseline at 5 minutes after local infiltration and at 30, 40, 50, 60, and 70 minutes after start of surgery in group $\mathrm{D}(p<0.05$; -Fig. 2B), while the magnitude of decrease was not significant from the baseline in group $P$.

On comparing the two groups, increase in HR after intubation was significantly blunted in group D. HR was consistently lower and controlled, but without bradycardia in group D ( - Fig. 2A). Similarly, MAP was lower, yet in the clinically desirable range of 75 to $85 \mathrm{~mm} \mathrm{Hg}$ in group D, whereas it was significantly higher in placebo group at $10,30,40$, and 50 minutes (-Fig. 2B).
The mean alveolar concentration (MAC) of isoflurane required in group $\mathrm{D}$ to maintain anesthesia was significantly less than that in placebo group (-Table 1). No significant difference was found in IBS between groups $\mathrm{P}$ and $\mathrm{D}$ (median $=1$ and $\mathrm{IQR}=0$ in both groups). Time to extubation, time to response to verbal commands, and orientation time were statistically comparable between the two groups ( $p>0.05$; - Table 2). The modified Aldrete's score and Ramsay's sedation score were similar in both the groups.

\section{Discussion}

The current study demonstrated that perioperative use of dexmedetomidine for transsphenoidal resection of pituitary tumors provide stable hemodynamics with decreased requirement of inhalational anesthetics, yet without delay in recovery parameters. The surgical field was satisfactory for carrying out the surgical procedure in all patients studied. Thus, dexmedetomidine does not seem to offer any advantage over placebo in surgical field.

Similar attenuation of rise in blood pressure in response to intubation with dexmedetomidine has been previously reported by Gopalakrishna et $\mathrm{al}^{6}$ and Kunisawa et $\mathrm{al}^{10}$. It is important to note here that we did not encounter bradycardia

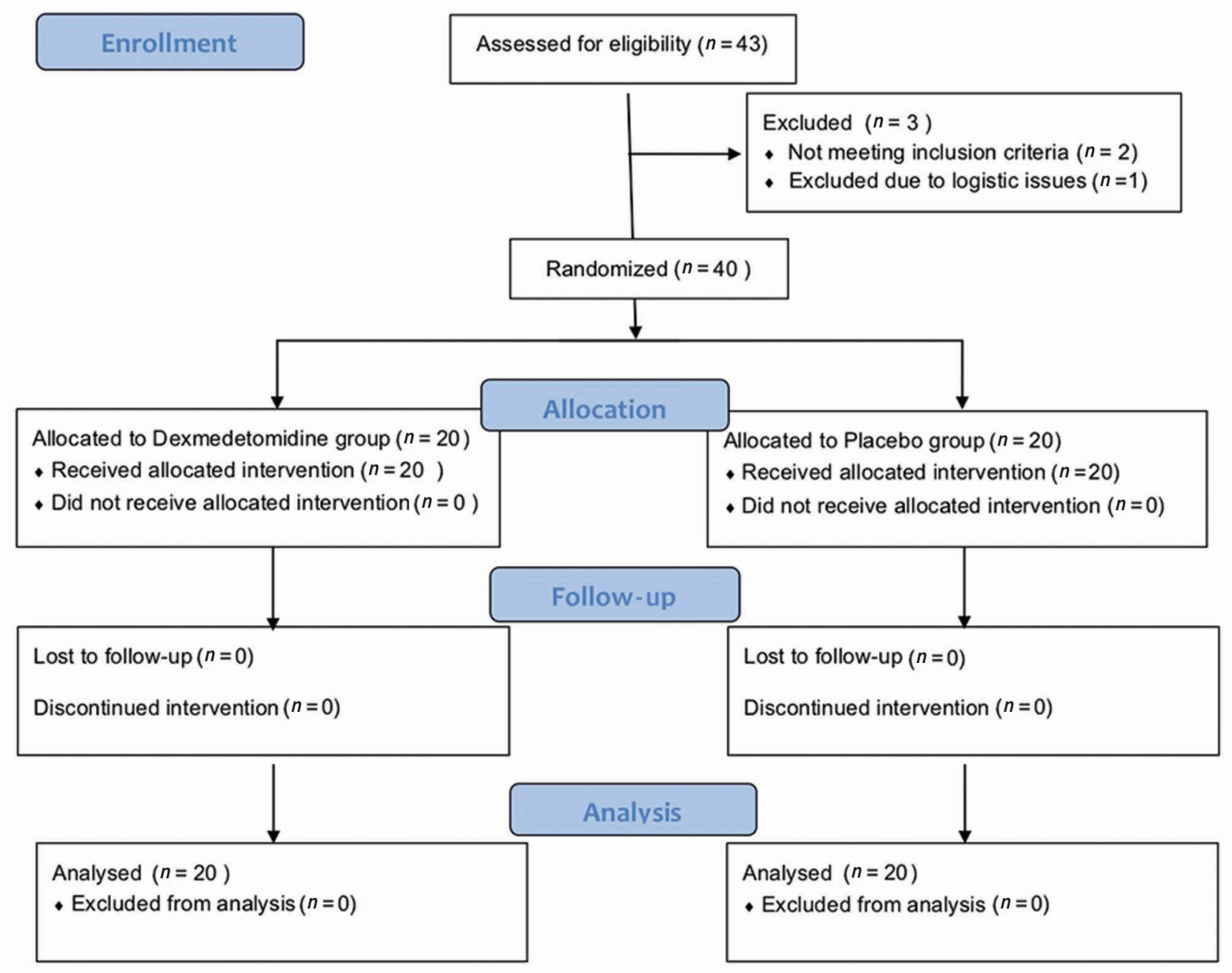

Fig. 1 Consolidated standards of reporting trials (CONSORT) flowchart showing patient selection flow. 

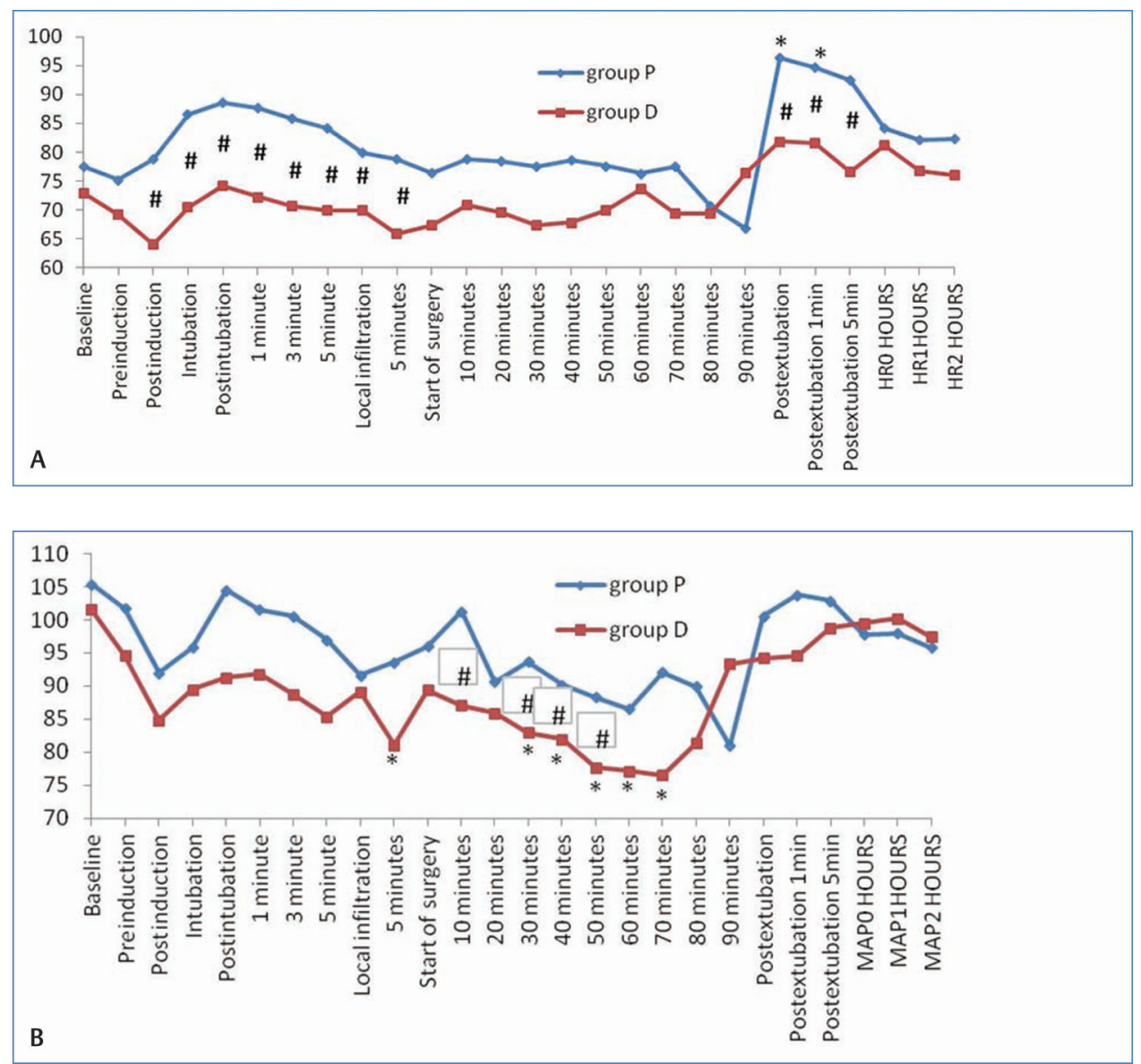

Fig. 2 (A) Variations in heart rate during anesthesia. P is placebo group, D is dexmedetomidine group, ${ }^{*}$ indicates $p<0.05$ on comparing with the baseline, \# indicates $p<0.05$ on comparing the two groups. (B) Variations in mean arterial pressure during anesthesia. $P$ is placebo group, $\mathrm{D}$ is dexmedetomidine group, ${ }^{*}$ indicates $p<0.05$ on comparing with the baseline, \# indicates $p<0.05$ on comparing the two groups.

Table 1 MAC at various time periods

\begin{tabular}{|l|l|l|l|}
\hline & Group P (mean \pm SD) & Group D (mean \pm SD) & $p$-Value \\
\hline MAC postinduction & $0.8 \pm 0.3$ & $0.8 \pm 0.2$ & 0.52 \\
\hline MAC intubation & $0.7 \pm 0.4$ & $0.6 \pm 0.3$ & 0.299 \\
\hline MAC Postintubation & $0.9 \pm 0.2$ & $0.8 \pm 0.2$ & $0.003^{\text {a }}$ \\
\hline MAC Local infiltration & $1.0 \pm 0.2$ & $0.8 \pm 0.2$ & $<0.001^{\text {a }}$ \\
\hline MAC start of surgery & $0.9 \pm 0.2$ & $0.8 \pm 0.2$ & $0.002^{\text {a }}$ \\
\hline MAC 10 minutes & $0.9 \pm 0.2$ & $0.8 \pm 0.1$ & $<0.001^{\text {a }}$ \\
\hline MAC 20 minutes & $1.0 \pm 0.2$ & $0.8 \pm 0.1$ & $<0.001^{\text {a }}$ \\
\hline MAC 30 minutes & $0.9 \pm 0.2$ & $0.8 \pm 0.2$ & $0.001^{\text {a }}$ \\
\hline MAC 60 minutes & $0.9 \pm 0.2$ & $0.7 \pm 0.2$ & $<0.001^{\text {a }}$ \\
\hline MAC 5 minutes prior to extubation & $0.8 \pm 0.3$ & $0.8 \pm 0.0$ & 1.00 \\
\hline
\end{tabular}

Abbreviations: MAC, minimum alveolar concentration; SD, standard deviation.

Note: aindicates $p$-Value $<0.05$ on comparing between the two groups, $\mathrm{P}$ is placebo group, $\mathrm{D}$ is dexmedetomidine group.

or hypotension during laryngoscopy and tracheal intubation as reported by Lawrence et al who used $2 \mu \mathrm{g} / \mathrm{kg}$ dexmedetomidine. ${ }^{11}$ So, the current study supports the use of low dose dexmedetomidine-1 $\mu \mathrm{g} / \mathrm{kg}$-to blunt cardiovascular response. During surgery, HR decreased by $15 \%$ and MAP decreased by $11 \%$ from baseline. This decrease in HR and BP were within normal physiological range, indicating stable hemodynamic profile with dexmedetomidine which is desirous in transsphenoidal pituitary surgery. However, better hemodynamic control did not translate into better bleeding scores in dexmedetomidine group akin to the findings of Gopalakrishna et al. ${ }^{6}$ The possibility of capillary bleeding which is less influenced by the hemodynamic profile during surgery may be a plausible explanation for this. 
Table 2 Recovery parameters

\begin{tabular}{|l|l|l|l|}
\hline Events & Group P (mean \pm SD) & Group D (mean \pm SD) & $p$-Value \\
\hline Time to extubation of trachea (min) & $11.6 \pm 3.3$ & $13.3 \pm 3.1$ & 0.09 \\
\hline Time to response to verbal commands (min) & $11.9 \pm 2.6$ & $13.5 \pm 2.9$ & 0.07 \\
\hline Orientation time (min) & $15.3 \pm 2.7$ & $16.5 \pm 3.2$ & 0.19 \\
\hline
\end{tabular}

Abbreviation: SD, standard deviation.

Note: $\mathrm{P}$ is placebo group, $\mathrm{D}$ is dexmedetomidine group.

The isoflurane requirement was lower in dexmedetomidine group to maintain BIS of 40 to 60 , similar to earlier reports using either isoflurane and sevoflurane. ${ }^{12-14}$ We also found similar recovery profile in dexmedetomidine and control groups, whereas Turgut et al had found longer times to extubation and orientation when dexmedetomidine was compared with IV remifentanil. ${ }^{15}$ However, Kim et al had found hemodynamically stable emergence with improved quality of recovery after nasal surgery with dexmedetomidine infusion. ${ }^{16}$ Our findings indicate that dexmedetomidine does not prolong recovery from anesthesia. The decreased requirement of inhalational agents while using dexmedetomidine infusion may contribute to faster recovery from anesthesia. Thus, it may be important to titrate anesthetic delivery to pharmacodynamic end-points such as BIS, while using dexmedetomidine to avoid excessive administration of inhalational agents.

The study has few limitations: (1). Blood loss was not quantified objectively and therefore, any benefit of better hemodynamics on bleeding may have been underestimated. (2). Majority of the included patients had pituitary macroadenoma, which tends to bleed less than more vascular tumors such as Cushing's disease.

In conclusion, dexmedetomidine $1 \mu \mathrm{g} / \mathrm{kg}$ followed by $0.5 \mu \mathrm{g} / \mathrm{kg} / \mathrm{h}$ infusion reduced hemodynamic response to intubation and provided stable intraoperative hemodynamics in patients undergoing transsphenoidal excision of pituitary tumor. The added advantages of dexmedetomidine include reduced intraoperative anesthetic requirement and a smooth recovery without undue delay.

\section{Conflict of Interest}

None declared.

\section{References}

1 Abou-Madi MN, Trop D, Barnes J. Aetiology and control of cardiovascular reactions during trans-sphenoidal resection of pituitary microadenomas. Can Anaesth Soc J 1980;27(5):491-495

2 Messick JM, Jr. Laws ER, Jr. Abboud CF. Anesthesia for transsphenoidal surgery of the hypophyseal region. Anesth Analg 1978;57(2):206-215
3 Muralidhar K, Bhanumurthy S. Attenuation of the cardiovascular responses to subcutaneous adrenaline in neurosurgical patients. Br J Anaesth 1992;68(3):264-267

4 Durmus M, But AK, Dogan Z, Yucel A, Miman MC, Ersoy MO. Effect of dexmedetomidine on bleeding during tympanoplasty or septorhinoplasty. Eur J Anaesthesiol 2007;24(5):447-453

5 Ayoglu H, Yapakci O, Ugur MB, et al. Effectiveness of dexmedetomidine in reducing bleeding during septoplasty and tympanoplasty operations. J Clin Anesth 2008;20(6):437-441

6 Gopalakrishna KN, Dash PK, Chatterjee N, Easwer HV, Ganesamoorthi A. Dexmedetomidine as an anaesthetic adjuvant in patients undergoing transsphenoidal resection of pituitary tumour. J Neurosurg Anesthesiol 2015;27(3):209-215

7 Salimi A, Sharifi G, Bahrani H, et al. Dexmedetomidine could enhance surgical satisfaction in trans-sphenoidal resection of pituitary adenoma. J Neurosurg Sci 2017;61(1):46-52

8 Aldrete JA. The post-anesthesia recovery score revisited. J Clin Anesth 1995;7(1):89-91

9 Ramsay MA, Savege TM, Simpson BR, Goodwin R. Controlled sedation with alphaxalone-alphadolone. BMJ 1974;2(5920):656-659

10 Kunisawa T, Nagata O, Nagashima M, et al. Dexmedetomidine suppresses the decrease in blood pressure during anesthetic induction and blunts the cardiovascular response to tracheal intubation. J Clin Anesth 2009;21(3):194-199

11 Lawrence CJ, Prinzen FW, de Lange S. The effect of dexmedetomidine on the balance of myocardial energy requirement and oxygen supply and demand. Anesth Analg 1996;82(3):544-550

12 Fragen RJ, Fitzgerald PC. Effect of dexmedetomidine on the minimum alveolar concentration (MAC) of sevoflurane in adults age 55 to 70 years. J Clin Anesth 1999;11(6):466-470

13 Aantaa R, Kanto J, Scheinin M, Kallio A, Scheinin H. Dexmedetomidine, an. $\alpha$ 2-adrenoceptor agonist, reduces anesthetic requirements for patients undergoing minor gynecologic surgery. Anesthesiology 1990;73(2):230-235

14 Kamibayashi T, Maze M. Clinical uses of alpha2-adrenergic agonists. Anesthesiology 2000;93(5):1345-1349

15 Turgut N, Turkmen A, Gökkaya S, Altan A, Hatiboglu MA. Dexmedetomidine-based versus fentanyl-based total intravenous anesthesia for lumbar laminectomy. Minerva Anestesiol 2008;74(9):469-474

16 Kim SY, Kim JM, Lee JH, Song BM, Koo BN. Efficacy of intraoperative dexmedetomidine infusion on emergence agitation and quality of recovery after nasal surgery. Br J Anaesth 2013;111(2):222-228 